\title{
Nonlinear-Embedding Design Methodology Oriented to LDMOS Power Amplifiers
}

\author{
Gianni Bosi, Member, IEEE, Antonio Raffo, Member, IEEE, \\ Francesco Trevisan, Valeria Vadalà, Member, IEEE, \\ Giovanni Crupi, Senior Member, IEEE, and Giorgio Vannini, Member, IEEE
}

\begin{abstract}
In this paper we apply for the first time the nonlinear-embedding technique to the design of power amplifiers based on laterally-diffused metal-oxide-semiconductor (LDMOS) field-effect transistors (FETs). Such a design technique is based on setting the transistor load line at the intrinsic current-generator plane, according to well-known theoretical guidelines. Then, the selected operating condition can be transposed at any design frequency at the extrinsic transistor terminals, by means of a model of the device nonidealities, such as the nonlinear intrinsic capacitances and the linear parasitic effects. A harmonicallytuned high-efficiency class-F and a wideband class- $A B$ power amplifiers operating within the FM broadcasting band $88 \div 108 \mathrm{MHz}$ based on a $10-\mathrm{W}$ LDMOS are then designed and realized. To definitely assess the validity of the proposed approach for the LDMOS technology, we compare the measured performance on the fabricated power amplifiers with the expected predictions.
\end{abstract}

Index Terms - Power amplifiers, power MOSFETs, power semiconductor devices, semiconductor device measurement, semiconductor device modeling.

\section{INTRODUCTION}

$\mathrm{P}$ OWER management is one of the most critical issues in modern electronic systems. Besides reducing the energy costs, an efficiency-oriented design translates itself into a simplified cooling system, a better component reliability, and therefore, a reduction of the maintenance costs.

In the majority of electronic apparatuses, apart from the power-supply system, the electronic elements that draw the greatest part of the energy are the power amplifiers (PAs). Today, their impact on the overall energy consumption of an electronic system is of paramount importance for any kind of application, like audio [1], [2], medical [3], radiofrequency (RF) [4], [5], and so on.

The use of operating classes based on a reduced conduction angle (e.g., class B and C) surely improves the efficiency, at the price of a reduction of other important parameters, such as linearity and power gain [6], [7]. New classes of operation [6]-[10] have been developed to increase the PA

Manuscript received July 28, 2017; revised October 26, 2017; accepted November 29, 2017. (Corresponding Author: Antonio Raffo.)

Gianni Bosi, Antonio Raffo, Francesco Trevisan, Valeria Vadalà, and Giorgio Vannini are with the Department of Engineering, University of Ferrara, 44122 Ferrara, Italy (e-mail: gianni.bosi@unife.it; antonio.raffo@unife.it; efficiency. They are mainly based on tuning the transistor harmonic terminations to shape the transistor waveforms and get two theoretical requirements:

- No overlap between the output current and voltage waveforms to reduce the power dissipated on the active device.

- $\quad$ No active power delivered at the harmonic frequencies.

If these conditions are both satisfied, a theoretical $100 \%$ efficiency is achievable [7]. As an example, the switching class-E PA is a very common choice for PA operating in the megahertz range, whereas for application at $\mathrm{RF}$ and microwaves, class-J [11] and class-F [12] operations are largely diffused together with some architectural implementations oriented to furtherly improve the efficiency [5], [13].

On the other hand, increasing the efficiency by harmonic manipulation typically implies conditions that may be harmful for the transistor reliability, in particular for the high electric fields induced by the peaks of the voltage waveforms [14], that have to be properly controlled to avoid any premature failure of the active devices [15]-[17].

Another drawback is related to the frequency selectivity of the matching networks, which is necessary when harmonic terminations need to be controlled. In Table I, we report some examples of state-of-art PAs. It is clear that for harmonicallytuned amplifiers, the efficiency becomes lower with respect to the theoretical values as larger design bandwidths are considered [18] because of the physical limitations of implementing the harmonic terminations over a wide range of frequencies. In some cases, the use of a reduced conduction angle condition (e.g., class- $\mathrm{AB}$ ) may be more convenient since tuning the matching networks only at the fundamental frequency reduces the complexity of the circuit, guaranteeing a sufficient performance over a large bandwidth.

From a theoretical point of view, the waveforms at the input and output port of the transistor in a PA are defined by considering the active device either as a switch (e.g., class E) or, more generally, as a controlled current generator. Indeed,

francesco.trevisan@unife.it;

valeria.vadala@unife.it; giorgio.vannini@unife.it).

Giovanni Crupi is with the Department of Biomedical and Dental Sciences and Morphofunctional Imaging, University of Messina, 98125 Messina, Italy (e-mail: crupig@unime.it). 
TABLE I

STATE-OF-ART POWER AMPLIFIERS

\begin{tabular}{|c|c|c|c|c|c|}
\hline Year & Technology & Class of Operation & Fractional Bandwidth & Output Power & Efficiency \\
\hline $2013[18]$ & GaN HEMT & $\mathrm{AB}$ & $58 \%$ & $100 \mathrm{~W}$ & $51 \div 61 \%$ \\
\hline 2014 [19] & LDMOS & $\mathrm{E}$ & $24 \%$ & $6.5 \mathrm{~W}$ & $>60 \%$ \\
\hline 2014 [20] & GaN HEMT & $\mathrm{F}$ & $0 \%$ & $7.7 \mathrm{~W}$ & $80.2 \%$ \\
\hline 2015 [21] & LDMOS & $\mathrm{E}$ & $20 \%$ & $>70 \mathrm{~W}$ & $82 \%$ \\
\hline 2016 [22] & GaN HEMT & $\mathrm{AB}$ & $27 \%$ & $4.9 \mathrm{~W}$ & $68 \%$ (PAE) \\
\hline 2016 [23] & GaN HEMT & $\mathrm{F}$ & $62 \%$ & $9 \div 11 \mathrm{~W}$ & $>60 \%$ \\
\hline 2017 [24] & GaN HEMT & $\mathrm{J}$ & $30 \%$ & $4 \div 4.7 \mathrm{~W}$ & $40 \div 50 \%$ (PAE) \\
\hline 2017 [25] & LDMOS & F-1 & $62 \%$ & $6.3 \div 10 \mathrm{~W}$ & $73 \div 79 \%$ \\
\hline \multirow[b]{2}{*}{ This work } & \multirow[b]{2}{*}{ LDMOS } & $\mathrm{AB}$ & $20 \%$ & $10 \mathrm{~W}$ & $55 \div 63 \%$ \\
\hline & & $\mathrm{F}$ & $0 \%$ & $8 \mathrm{~W}$ & $76 \%$ \\
\hline
\end{tabular}

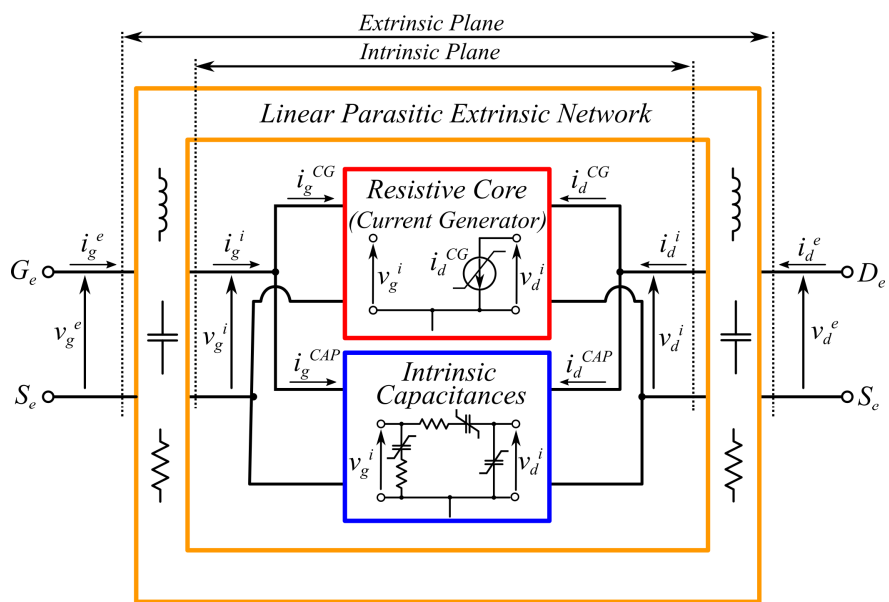

Fig. 1. Topology of a nonlinear model for an LDMOS device.

this is a strong simplification, because in an actual device, nonlinear intrinsic capacitances and linear parasitic effects alter the ideal device behavior and, as a consequence, hide the transistor reference plane where the proper waveforms should be implemented. Designers can deal with these effects by using some simplified models. As an example, for a class-E design, the capacitive behavior of the output port is typically modeled as either a linear or a nonlinear capacitor [15], [16], [21], according to the level of accuracy the designer wants to achieve.

In this paper, we discuss a general methodology for the design of laterally-diffused metal-oxide-semiconductor (LDMOS) field-effect transistor (FET) PAs, which exploits the waveforms measured at the current-generator plane as the starting point. Successively, by using a model of the device nonlinear capacitances and parasitic elements, we can embed their contributions and get the proper terminations at the actual device terminals. This technique is referred as nonlinear embedding [26], since in the most general case such models are nonlinear. Although already presented within the framework of microwave applications, we want to extend this methodology to a different kind of devices and applications, showing how it can be successfully adopted also in the field of power electronics.

With respect to conventional approaches, the nonlinearembedding methodology does not require extensive, timeconsuming load/source-pull measurements or simulations for identifying the device optimum operating condition. This is due to the fact that the optimum condition, that is univocally defined by the power-amplifier design theory for each class of operation [7], represents the starting point of the nonlinear-embedding approach. We will use this approach for the design of two different PAs oriented to FM broadcasting systems. The first PA is targeted to operate under a high-efficiency condition by using harmonic manipulation. The design of the second PA is targeted to achieve a wideband performance. Both design examples put in evidence the capabilities of the nonlinearembedding methodology. We will also compare this design approach with conventional ones which exploit a simplified linear model of the intrinsic capacitances showing how the nonlinear-embedding methodology allows more accurate results.

Apart from being of great interest for researchers because of its continuous improvements [27]-[29], the choice of investigating the LDMOS technology is mainly related to its large diffusion in the telecommunication systems for operating frequencies up to some gigahertz [30]-[32]. Nevertheless, thanks to its generality, the nonlinear-embedding technique can be also applied to very different transistor technologies [33], [34].

\section{THE NONLINEAR-EMBEDDING APPROACH}

The theory of PA design [6], [7] assumes the transistor as a controlled nonlinear current generator, where, for an FET, the controlling variables are the input (gate) and output (drain) voltages according to well-known algebraic relationships [35][37]. This is clearly a simplistic approximation of the transistor behavior, which works well for a preliminary theoretical analysis but has severe limitations. To explain better this aspect, we can look at the general topology for an LDMOS-transistor model depicted in Fig. 1. Apart from the current generator, which is part of the so-called resistive core, as the operating frequency increases, we have to take into account the contribution of some intrinsic nonlinear dynamic effects. They are typically modeled as nonlinear capacitances [38] connected in parallel with the current generator, although some series resistors may also be included for improving the model predictions at the very end of the device bandwidth [39]. The current generator together with the capacitive network constitute the intrinsic device for which we define the intrinsic reference plane. To get to the actual device terminals, the so- 
called extrinsic reference plane, there exist some structures (bonding wires, package, etc.), that introduce linear parasitic effects that must be also considered. All these elements tend to hide the device current generator as the operating frequency increases, making it practically impossible to directly control and obtain the desired waveforms at its terminals, as defined by the design theory. This aspect may become of crucial importance, especially when harmonic tuning has to be performed [40].

The nonlinear-embedding approach starts from the waveforms at the current-generator plane corresponding to the specific operating condition of interest. In the frequency domain, we can define them as

$$
\begin{aligned}
& V^{i}\left(k \omega_{H F}\right)=\left[V_{g}^{i}\left(k \omega_{H F}\right), V_{d}^{i}\left(k \omega_{H F}\right)\right] \quad k=0,1,2, \ldots, N \\
& I^{C G}\left(k \omega_{H F}\right)=\left[I_{g}^{C G}\left(k \omega_{H F}\right), I_{d}^{C G}\left(k \omega_{H F}\right)\right]
\end{aligned}
$$

where $\omega_{H F}=2 \pi f_{H F}$ is the design frequency and $N$ is a suitable number of harmonics sufficient for an accurate description of the waveforms. It should be noticed that, in principle, since the current generator is a frequency-independent element, we can achieve the waveforms in (1) at any frequency in terms of amplitudes and phases of their spectral components. However, they will correspond to different waveforms at the extrinsic plane, owing to the contributions of the nonlinear intrinsic capacitances and the linear parasitic elements at the selected $\omega_{H F}$. As a consequence, the waveforms at the current-generator plane must be shifted at the extrinsic plane accounting for those contributions.

If models of the intrinsic capacitances and of the parasitic network are available, we can derive the corresponding waveforms at the extrinsic plane following these steps, summarized in Fig. 2:

1) Accordingly with Fig. 1, we assume the intrinsic capacitances in parallel with the resistive-core that includes the current generator, and thus subject to the same voltage phasors in (1). Therefore, we can use the capacitance model to calculate the displacement current contribution at $\omega_{H F}$ (and its harmonics) for the specific operating regime selected at the current-generator plane, i.e.,

$$
I^{C A P}\left(k \omega_{H F}\right)=\left[I_{g}^{C A P}\left(k \omega_{H F}\right), I_{d}^{C A P}\left(k \omega_{H F}\right)\right], \quad k=0,1,2, . ., N
$$

2) The total intrinsic current can now be calculated as the sum of the resistive-core (i.e., current generator) and the capacitive contributions, i.e.,

$$
I^{i}\left(k \omega_{H F}\right)=I^{C G}\left(k \omega_{H F}\right)+I^{C A P}\left(k \omega_{H F}\right) \quad k=0,1,2, \ldots, N
$$

3) By using the parasitic-element descriptions, we can now shift the current and the voltage waveforms from the intrinsic to the extrinsic plane, thus obtaining

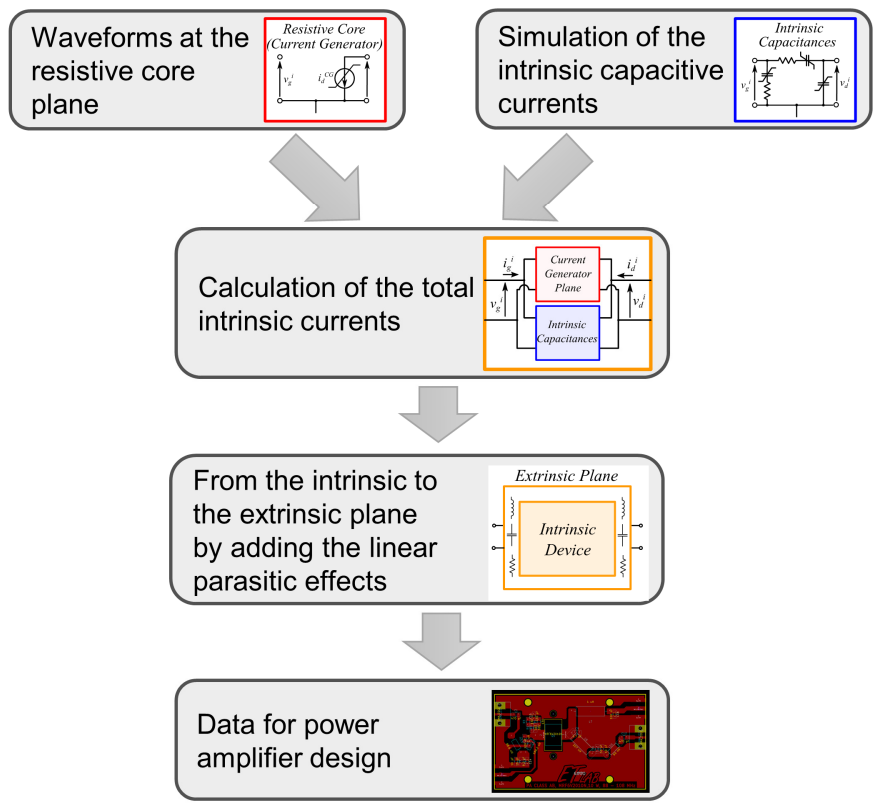

Fig. 2. Flowchart of the nonlinear-embedding methodology.

$$
\begin{aligned}
& V^{e}\left(k \omega_{H F}\right)=\left[V_{g}^{e}\left(k \omega_{H F}\right), V_{d}^{e}\left(k \omega_{H F}\right)\right] \\
& I^{e}\left(k \omega_{H F}\right)=\left[I_{g}^{e}\left(k \omega_{H F}\right), I_{d}^{e}\left(k \omega_{H F}\right)\right]
\end{aligned}
$$

These quantities correspond, in the frequency domain, to the time-domain waveforms we must guarantee at the accessible extrinsic terminals of the device to impose the selected operating condition at the current-generator plane.

From the extrinsic current and voltage phasors, we can get all the related data, such as the performance (e.g., output power, efficiency, etc.), together with the required input and output terminations at both the fundamental frequency and harmonics. For example, the load terminations will be given by

$Z_{L}^{e}=-\frac{V_{d}^{e}\left(k \omega_{H F}\right)}{I_{d}^{e}\left(k \omega_{H F}\right)} \quad k=1,2, \ldots, N$

and the input impedance of the device, which can be used for the design of the input matching network [41], is

$Z_{i n}^{e}=\frac{V_{g}^{e}\left(k \omega_{H F}\right)}{I_{g}^{e}\left(k \omega_{H F}\right)} \quad k=1,2, \ldots, N$

The whole procedure may be limited by some pre-matching networks included in the packaged device to ease the matchingnetwork design for very high-power transistors. This typically has the drawback of limiting the device bandwidth and, as a consequence, the capability of externally tuning the harmonic terminations. However, if the pre-matching circuit is known, the nonlinear-embedding approach could be still applied by including it in the parasitic-network model for assessing the fundamental termination to be synthesized at the device plane. 


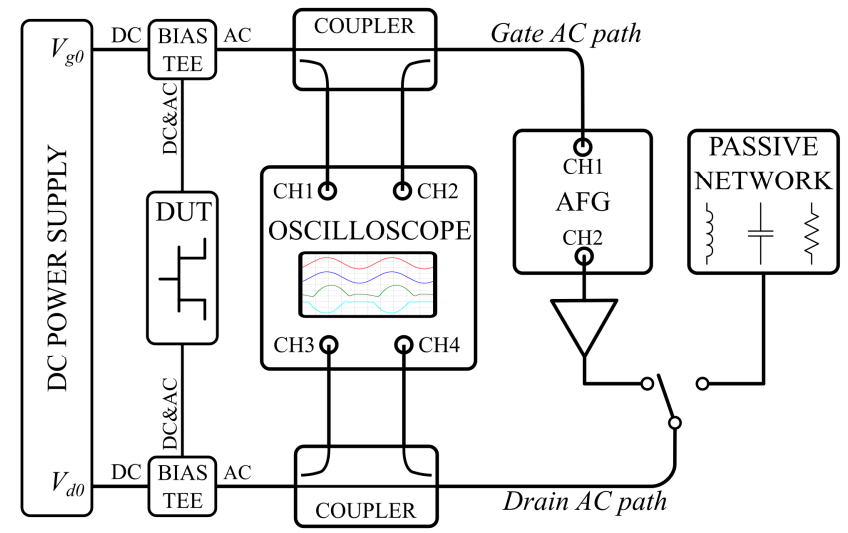

Fig. 3. Block diagram of the low-frequency measurement setup implementing the time-domain active load-pull system.

\section{THE Characterization SetuP}

The time-domain waveforms at the current-generator plane, corresponding to the selected operating condition, are the first data we need to define for applying the nonlinear-embedding procedure. We might exploit a model of the device current generator [34], perform some simulations in a CAD environment, and get the desired time-domain voltage and current waveforms. In this case, the accuracy of the model is a crucial aspect, especially when dealing with devices strongly affected by thermal effects as large-periphery LDMOS transistors. These effects can significantly alter the dynamic behavior of the current generator with respect to the conventional DC I/V description, typically assumed as a reference. This issue is further emphasized when new transistor technologies are investigated [42], for which the accurate modeling of the current generator is still an open research problem because of complex nonlinear phenomena affecting these devices.

A valuable alternative to simulations is to directly measure the current-generator behavior for the load line that meets the design requirements. As stated in Section II, this cannot be immediate at high frequency because of the linear and nonlinear reactive contributions due to the intrinsic capacitances and other parasitic elements. However, this problem can be overcome by measuring the device at lower frequencies. To this end we have developed the setup shown in Fig. 3 [43]. It is a time-domain load-pull system that performs an active synthesis of the device terminations at both the fundamental frequency and harmonics. The operating condition for the device under test (DUT) is imposed by injecting two incident waves at its input and output ports. By means of two dual-directional couplers connected to a $4-\mathrm{GHz}$ oscilloscope, we can monitor both the incident and reflected waves, whose combination defines the voltage and current waveforms at the DUT plane. The injected signals are provided by means of an arbitrary function generator (AFG), which allows one to control not only the fundamental components but also the harmonic ones. On the drain path, an amplifier may be inserted when the output power levels are too high to be handled by the AFG only. Alternatively to the active synthesis of the load, the output port of the DUT can be terminated by a passive network properly designed to implement the desired load conditions. The bias condition is imposed by a DC power supply and coupled with the AC signals by means of two bias tees. The actual implementation of the system allows controlling the incident waves up to $120 \mathrm{MHz}$ under multi-harmonic regime, whereas the setup bandwidth is limited by the couplers at $400 \mathrm{MHz}$. The correction of the measurements for the cable losses and phase shifts is implemented by using a characterization of the signal paths based on their S-parameter measurements. This procedure allows shifting the acquired data from the oscilloscope to the DUT plane [43].

For the characterization of the current generator, in order to get data consistent with the RF operation, we need to select a frequency $\omega_{L F}=2 \pi f_{L F}$ above the thermal-phenomena cut-off, for which a value of $1 \mathrm{MHz}$ is commonly accepted [35]. Such a cut-off frequency is also suitable for taking into account deeplevel trapping effects, which, although not affecting the $\mathrm{Si}$ LDMOS performance, strongly influence the transistor behavior in more recent technologies.

In addition, the characterization frequency has to be sufficiently low to neglect any contribution from the reactive effects of the device. Under this condition, the measured data refer to the current-generator plane only. Indeed, ohmic parasitic effects due to the access structures (i.e., package flanges, bonding wires, transmission lines, etc.) do exist, although they can be easily de-embedded from the measured data.

Once the desired load line is measured, the thermal and trapoccupation states are fixed and the $\mathrm{I} / \mathrm{V}$ relationship will not change with frequency as long as it is measured above the lowfrequency cut-off. Moreover, there should exist a condition at the extrinsic plane for any higher frequency $\omega_{H F}=2 \pi f_{H F}$ such that the current and voltage waveforms at the current-generator plane correspond to the measured ones at $\omega_{L F}$. Such a condition can be investigated by exploiting the nonlinear-embedding procedure, using as a starting point the low-frequency measured phasors, whose spectra are rigidly shifted from $\omega_{L F}$ to $\omega_{H F}$, i.e.,

$\begin{aligned} & V^{C G}\left(k \omega_{L F}\right) \\ & I^{C G}\left(k \omega_{L F}\right)\end{aligned} \stackrel{\text { fromLF to } H F}{\longrightarrow} \begin{aligned} & V^{C G}\left(k \omega_{H F}\right) \\ & I^{C G}\left(k \omega_{H F}\right)\end{aligned} \quad k=0,1,2, \ldots, N$

It is worth noticing that, in principle, the whole procedure is frequency independent, so that we can transpose the measured data to any design frequency that allows for waveform harmonic control at the intrinsic current-generator plane. This feature becomes extremely useful when wideband circuits need to be designed; the only limitation is related to the accuracy of the available parasitic and capacitance models in the bandwidth of interest.

\section{Design of Two Power Amplifiers}

We now discuss the design of two PAs based on the 10-W LDMOS MRF6V2010N by Freescale, which can operate within the band $10 \div 450 \mathrm{MHz}$, covering the FM broadcasting 


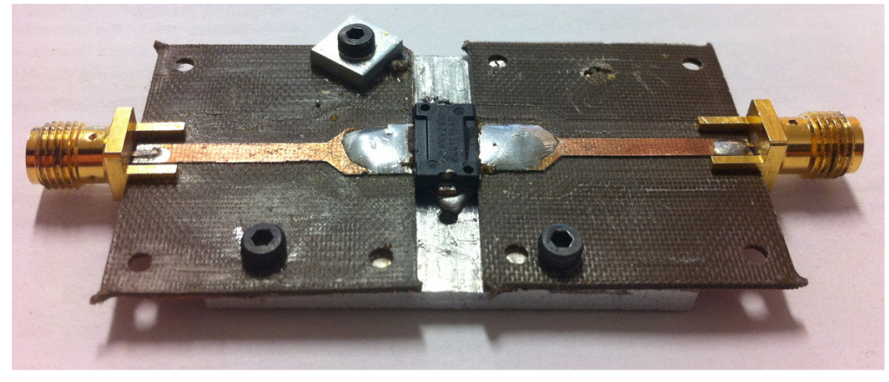

Fig. 4. LDMOS transistor mounted on the PCB board for the low-frequency characterization.

band we are interested to, i.e., $88 \div 108 \mathrm{MHz}$.

The aim of the two designs is to point out the capabilities of the nonlinear-embedding methodology, by selecting two different operating conditions. For the first PA, we focused on the maximization of its efficiency, keeping the output power at a sufficiently high level under continuous-wave regime. In this case, tuning the load only at the fundamental frequency is not sufficient, and we needed to properly control also the harmonics [17], [30]. Such a kind of evaluation may be easily performed by exploiting the setup described in Section III. The availability of an AFG as a signal source allows controlling also the harmonic components of the signals injected at the DUT ports. Harmonic tuning tends to limit the PA bandwidth because of the selectivity required for the matching networks. Therefore, as a second design, we considered a wideband PA. In this case, only the load impedance at the fundamental frequency is tuned and the nonlinear-embedding approach is used to derive the transistor terminations within the whole FM broadcasting band.

To connect the transistor to the setup, we mounted it on a customized PCB board fabricated on a Diclad 527 substrate (Fig. 4). The device gate and drain flanges are soldered to two $50-\Omega$ microstrip lines reaching the external SMA connectors. The characteristics of the lines have been designed to have negligible influence at low frequency (i.e., $2 \mathrm{MHz}$ ).

The transistor was preliminarily characterized by means of DC I/V conventional measurements for evaluating the best operating point for the device and a first estimation of its performance. Once the bias point was selected, i.e., $V_{g 0}=2.4 \mathrm{~V}$, $V_{d 0}=42.5 \mathrm{~V}, I_{d 0}=20 \mathrm{~mA}$, we identified the load lines corresponding to the two operations of interest, which are reported in Figs. 5 and 6, together with the output waveforms. The high-efficiency load line (Fig. 5(a)) was obtained by actively synthesizing the load impedances reported in Table II. They realize a class-F operation, which allows a higher output power than other conventional classes of operation with a reduced conduction angle (e.g., class C). In particular, with this load line, we achieved an RF output power of $8.5 \mathrm{~W}$ with a drain efficiency of $86.7 \%$. By looking at the measured waveforms in Fig. 5(b), we can see that their shapes are consistent with the theoretical ones, i.e., a square wave for the voltage and a halftruncated sinusoid for the current, with a minimum superposition. In addition, we can easily monitor if the selected dynamic condition may affect the transistor reliability by comparing the data with the device maximum ratings. As an example, the maximum drain voltage allowed for this device is

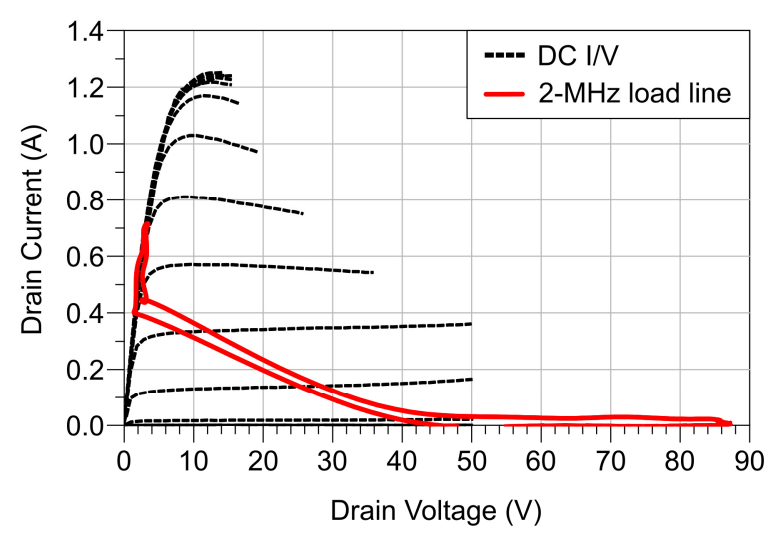

(a)

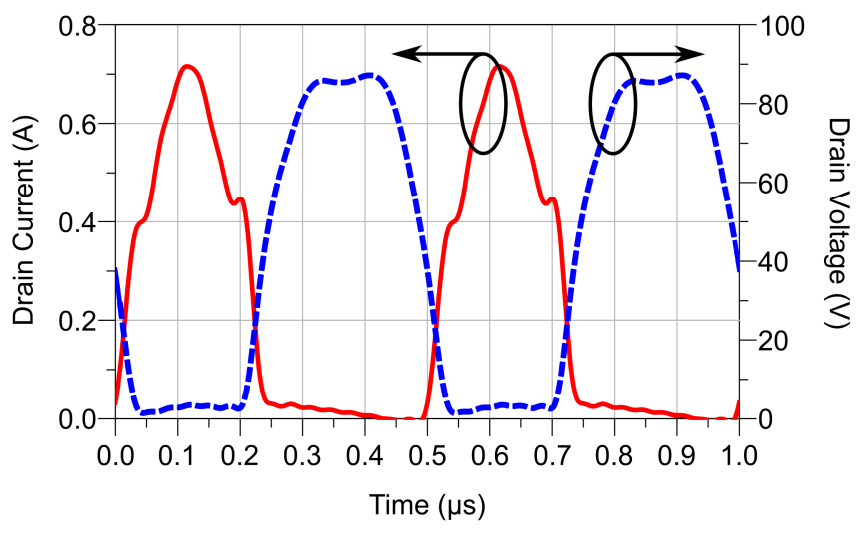

(b)

Fig. 5 High-efficiency class-F load line synthesized at $2 \mathrm{MHz}$ superimposed to the DC I/V characteristics (a) and corresponding waveforms (b).

$110 \mathrm{~V}$, whereas the load line highlights a maximum dynamic value of approximately $88 \mathrm{~V}$, which is sufficiently low to avoid any reliability issue.

We show the class-AB load line under saturated output power operation and the corresponding waveforms in Fig. 6. As reported in Table II, the impedances at harmonics were not set, focusing only on the fundamental one. Nevertheless, their values were controlled in order to keep them sufficiently low to avoid a strong influence on the PA performance. The measured performance in this case was $9.4 \mathrm{~W}$ with a drain efficiency of $68.2 \%$, which is consistent with the selected class of operation. It is also evident that the absence of harmonic tuning keeps the maximum value of the drain voltage lower than the class-F load line.

To apply the nonlinear-embedding procedure, we first need models of both the intrinsic capacitances and the extrinsic parasitic elements. In this particular case, we identified these models from multi-bias S-parameter measurements [44], [45]. The parasitic network has been identified by means of cold-FET data by using a conventional approach [46]. Then, by deembedding the parasitic effects from the S-parameters, the intrinsic capacitive network can be easily determined and implemented as a look-up-table model for direct nonlinear simulations [38], [46]. Indeed, the use of a customized model is not mandatory. Alternative solutions, such as the use of 


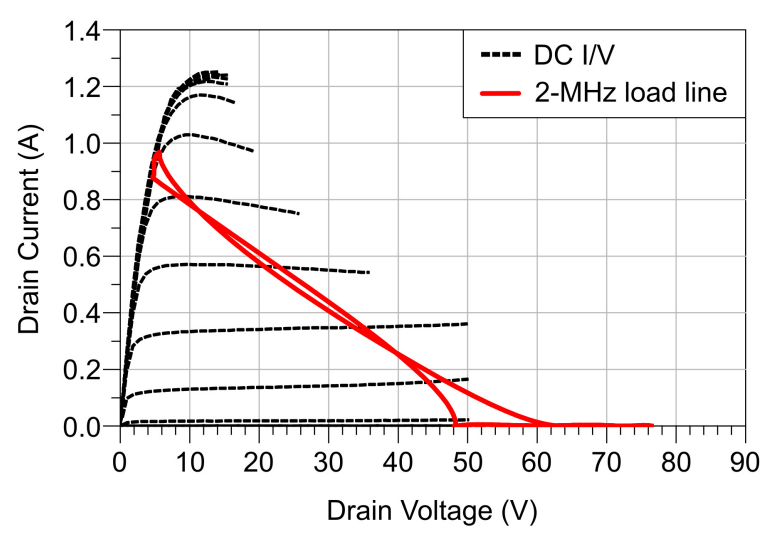

(a)

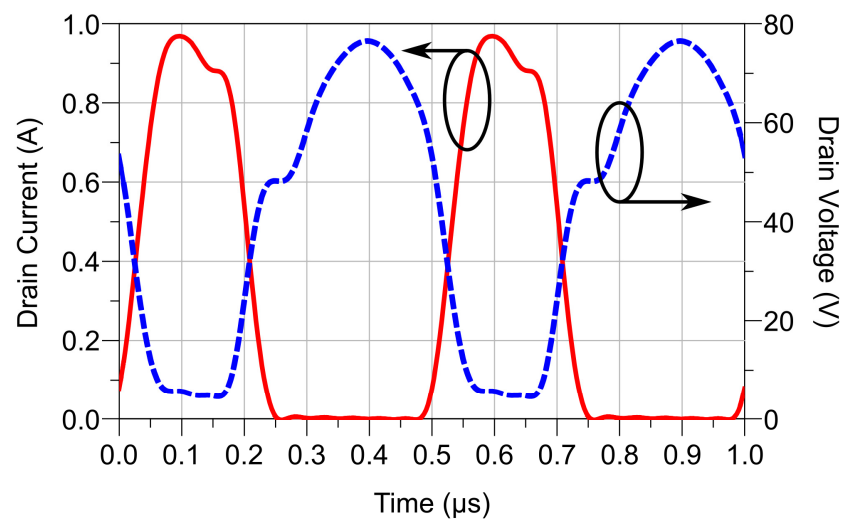

(b)

Fig. 6 Class-AB load line synthesized at $2 \mathrm{MHz}$ superimposed to the DC I/V characteristics (a) and corresponding waveforms (b).

TABLE II

LOAD IMPEDANCES CORRESPONDING TO THE LOW-FREQUENCY LOAD LINES

\begin{tabular}{ccc}
\hline \hline Frequency & $\begin{array}{c}\text { Class-F Load } \\
\text { Impedance }\end{array}$ & $\begin{array}{c}\text { Class-AB Load } \\
\text { Impedance }\end{array}$ \\
\hline $2 \mathrm{MHz}$ & $145.6+\mathrm{j} 6.5 \Omega$ & $73-\mathrm{j} 10 \Omega$ \\
$4 \mathrm{MHz}$ & $0+\mathrm{j} 6.2 \Omega$ & $30+\mathrm{j} 17 \Omega$ \\
$6 \mathrm{MHz}$ & $1.3+\mathrm{j} 1.0 \mathrm{k} \Omega$ & $39+\mathrm{j} 26 \Omega$ \\
\hline \hline
\end{tabular}

manufacturer models, could be adopted, provided that the description of the intrinsic capacitances and the extrinsic parasitic elements are available.

As a next step, we transposed the low-frequency load lines of the two classes of operation, to the design frequency by exploiting the nonlinear-embedding procedure described in Section II. For the high-efficiency class-F condition, we considered a design frequency of $100 \mathrm{MHz}$. In Table III we report the load and source impedances predicted by the nonlinear-embedding methodology and used for the synthesis of the PA matching networks. Since harmonic tuning was performed, we list the first three harmonics.

Regarding the class-AB load line, we applied the nonlinear embedding from 88 to $108 \mathrm{MHz}$ to get the design data for a wideband PA. For the sake of brevity, we report in Table IV the source and load impedances just at the edges and at the center of the selected FM bandwidth. It is worth noticing how, differently from conventional approaches, the impedance

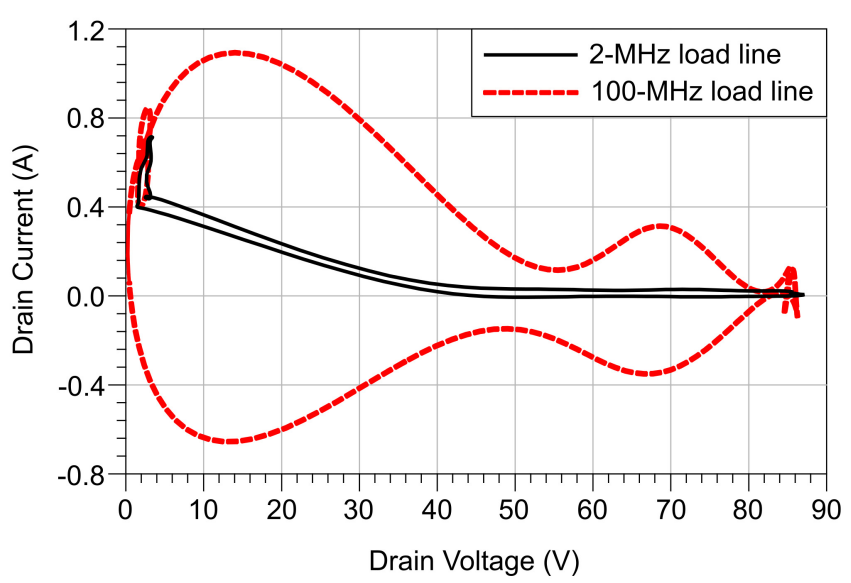

(a)

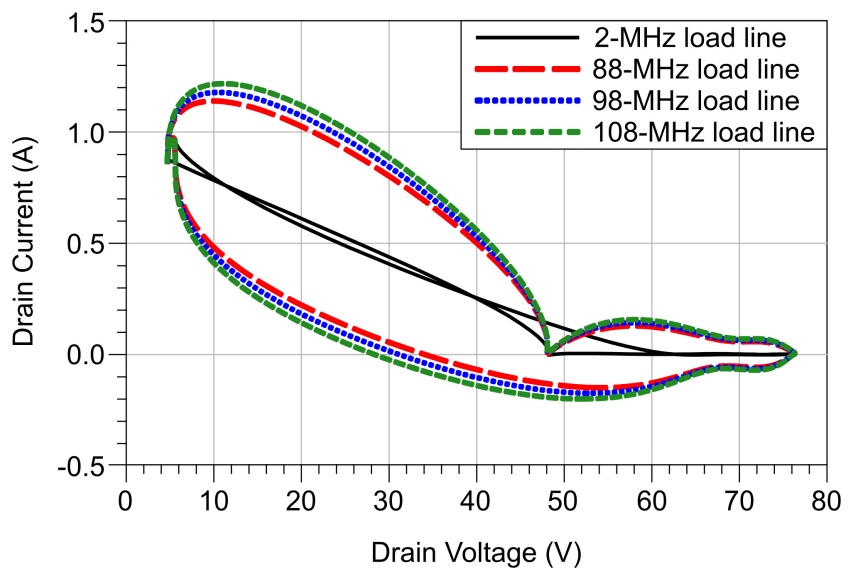

(b)

Fig. 7 Low-frequency load lines (solid lines) and predicted load lines at design frequency (dashed lines) for the high-efficiency class-F (a) and the wideband class-AB (b) conditions.

TABLE III

LOAD/SOURCE IMPEDANCES AT THE DESIGN FREQUENCY FOR THE CLASS-F LOAD LINE

\begin{tabular}{ccc}
\hline \hline Frequency & Load Impedances & Source Impedances \\
& & \\
\hline $100 \mathrm{MHz}$ & $77.8+\mathrm{j} 71.1 \Omega$ & $9.1+\mathrm{j} 72.6 \Omega$ \\
$200 \mathrm{MHz}$ & $\mathrm{j} 2.0 \Omega$ & $-\mathrm{j} 2.1 \Omega$ \\
$300 \mathrm{MHz}$ & $\mathrm{j} 59.7 \Omega$ & $\mathrm{j} 1.8 \Omega$ \\
\hline \hline
\end{tabular}

TABLE IV

LOAD/SOURCE IMPEDANCES AT THE DESIGN FREQUENCIES FOR THE CLASS-AB LOAD LINE

\begin{tabular}{ccc}
\hline \hline Frequency & Load Impedances & Source Impedances \\
\hline $88 \mathrm{MHz}$ & $69.7+\mathrm{j} 17.5 \Omega$ & $14.1+\mathrm{j} 94.5 \Omega$ \\
$98 \mathrm{MHz}$ & $68.2+\mathrm{j} 20.1 \Omega$ & $13.7+\mathrm{j} 84.8 \Omega$ \\
$108 \mathrm{MHz}$ & $66.5+\mathrm{j} 22.5 \Omega$ & $13.3+\mathrm{j} 76.9 \Omega$ \\
\hline \hline
\end{tabular}

trajectory to be synthesized can be simply retrieved by applying (2)-(5) in the entire bandwidth, avoiding time-consuming optimization procedures.

In Fig. 7 we report the predictions of the load lines at the extrinsic plane compared with the measurements at the currentgenerator plane. It is evident how the latter are masked by the 


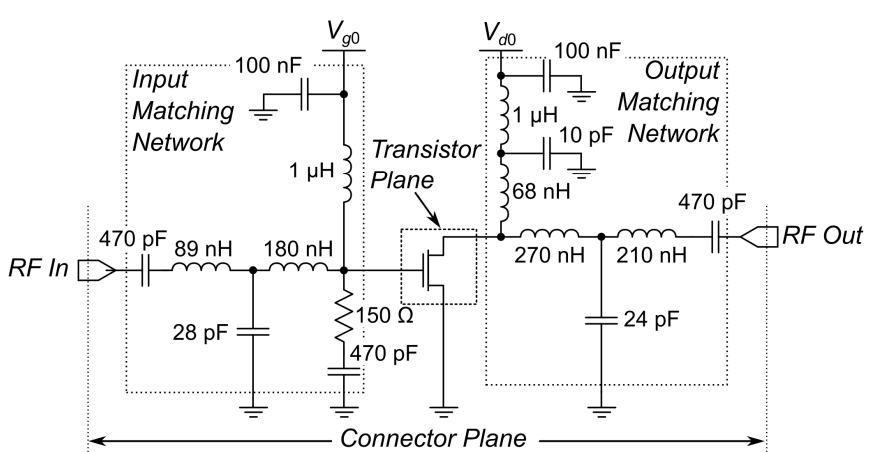

(a)

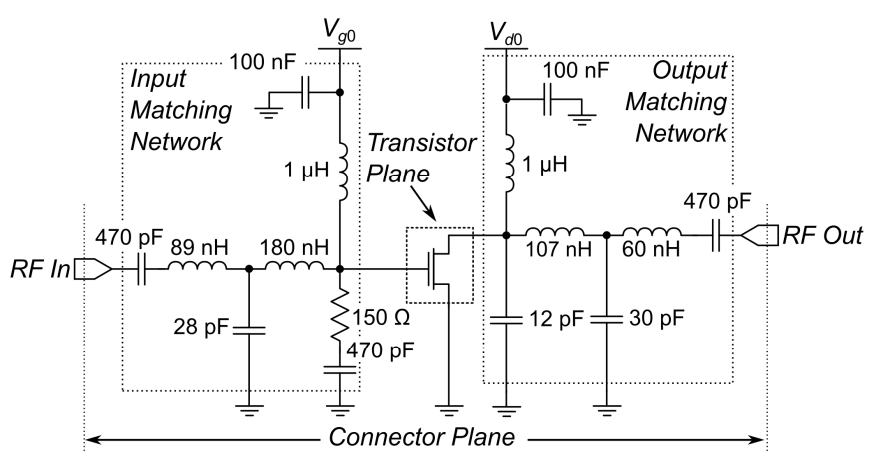

(b)

Fig. 8 Schematics of the class-F (a) and class-AB (b) fabricated PAs with the indication of the connector and transistor planes.

contributions of the reactive elements of the device. In Fig. 7(b), it is also evident a slight variation of the extrinsic load lines with frequency, which reflects the values of the load impedances reported in Table IV.

\section{POWER AMPLIFIER FABricATION}

In Fig. 8 we report the schematics implemented for the fabrication of the PAs, where the matching and bias networks have been designed to synthesize the impedances derived from the nonlinear-embedding procedure.

The output-matching networks are quite complex in both cases, although for different reasons: the need for harmonic control in the high-efficiency class-F PA and the wideband requirement for the class- $\mathrm{AB} \mathrm{PA}$.

Regarding the input network, the $\mathrm{R}-\mathrm{C}$ shunt has been introduced for stability issues in both cases. Moreover, to guarantee adequate matching also under small-signal operation, we realized a trade-off between the source impedance values in Table III and IV and the ones, derived from S-parameter measurements at the selected bias point, that maximize the small-signal matching over the bandwidth $88 \div 108 \mathrm{MHz}$. The same L-C-L input matching network was sufficient to get good performance over the required bandwidth in both the PAs, as will be shown in the following.

The circuits were fabricated on a conventional FR-4 substrate, and are reported in Fig. 9.

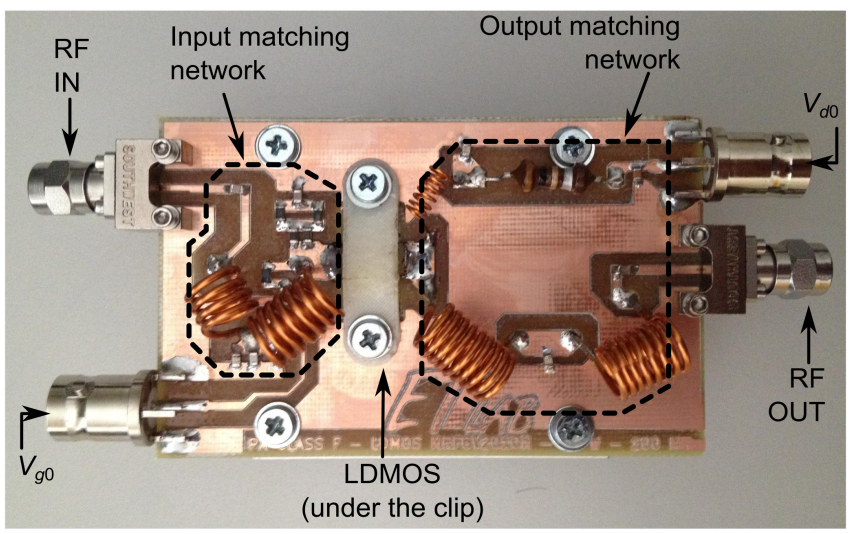

(a)

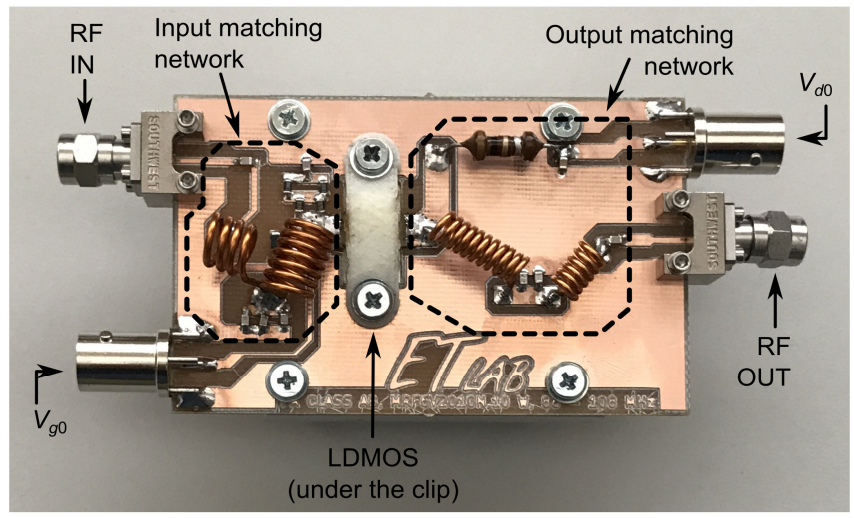

(b)

Fig. 9 Photos of the fabricated PAs: class F (a) and class AB (b).

\section{POWER AMPLifier VALIDATION}

\section{A. Small-signal verification}

We first measured the small-signal behavior of the PAs for their nominal bias point, i.e., $V_{g 0}=2.4 \mathrm{~V}, V_{d 0}=42.5 \mathrm{~V}$, by using a vector network analyzer. We show the results in terms of input matching and small-signal gain in Fig. 10. In the bandwidth for which the input matching network was designed, we obtained an input matching better than $-14 \mathrm{~dB}$ and $-16 \mathrm{~dB}$ for the class-F and the class- $A B$ PAs respectively. The small-signal gain of the class-F PA shows a large variability in frequency related to the selectivity of the output-matching network. We expected the peak gain not lying at the design frequency, since no specification has been considered for this parameter in the design phase, giving priority to the output power and efficiency under large-signal operation. On the contrary, the class-AB amplifier, for which the matching networks were optimized within the whole FM bandwidth, has an approximately flat gain, with a variability limited to $0.5 \mathrm{~dB}$.

\section{B. Large-signal measurements}

The next step was to verify the PAs performance under actual operation, i.e., large-signal conditions. To this aim, we exploited a simplified version of the setup described in Section III, removing the bias-tees (which are included in the fabricated PAs) and terminating the output port on a constant $50-\Omega$ load. 


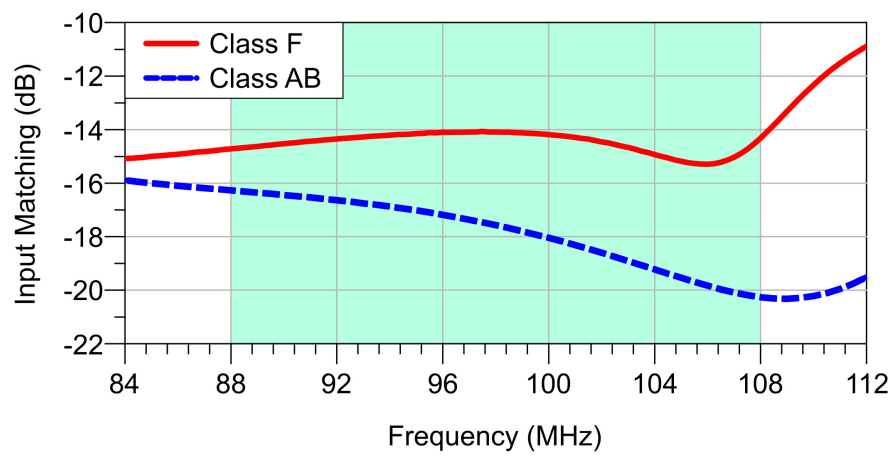

(a)

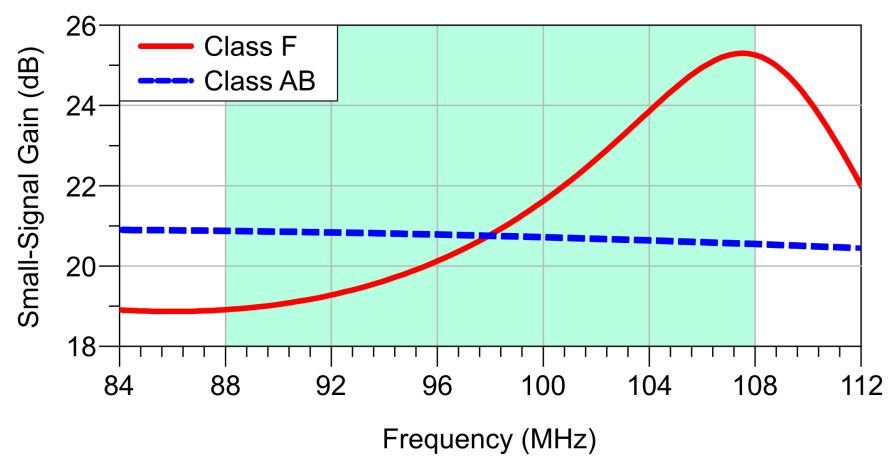

(b)

Fig. 10 Input matching (a) and small-signal gain (b) of the fabricated PAs. The shaded areas highlight the bandwidth $88 \div 108 \mathrm{MHz}$.

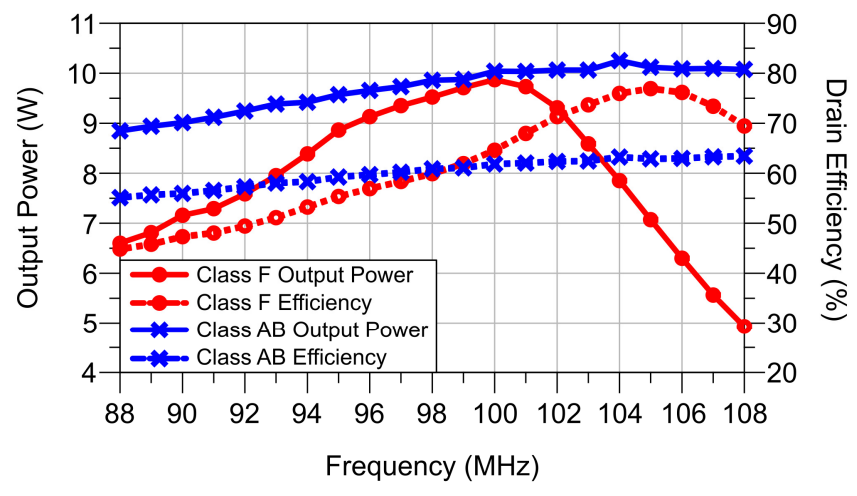

Fig. 11 Measured output power (solid red line) and drain efficiency (dotted blue line) for an input power of $14.4 \mathrm{dBm}$ (class F) and $16.9 \mathrm{dBm}$ (class $\mathrm{AB}$ ) over the bandwidth $88 \div 108 \mathrm{MHz}$. Data refers to the connector plane (Fig. 8).

We first measured the PAs performance for a constant input power level over the bandwidth $88 \div 108 \mathrm{MHz}$. The results are reported in Fig. 11. The class-F PA achieves a maximum output power at $100 \mathrm{MHz}$, whereas the maximum efficiency is reached at $105 \mathrm{MHz}$. The class-AB PA performance is instead more constant over frequency, consistently with its wideband design. As expected from this class of operation and for the selected design condition, it delivers higher power with a lower efficiency.

In both cases the performance is lower than the designed one. This is indeed an expected result since the measurements include the losses of the matching networks, which lower the overall efficiency. On the contrary, the design data are referred to the transistor extrinsic reference plane. Therefore, for a fair

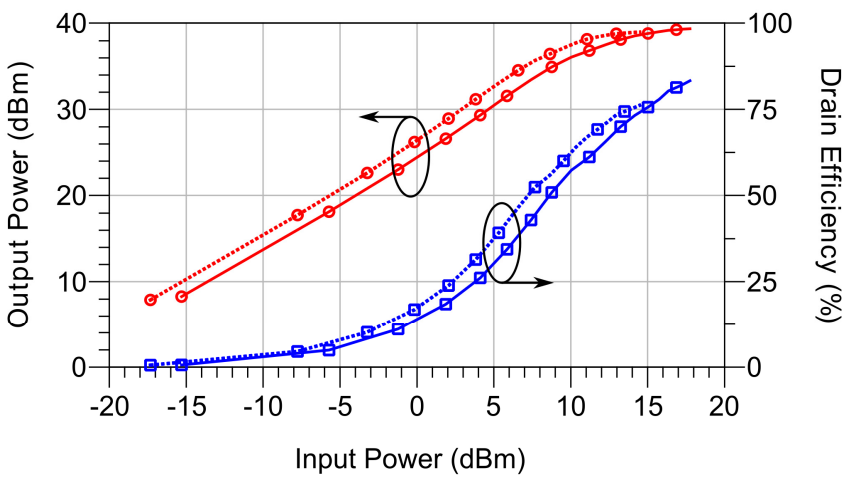

Fig. 12 Output power (red circled lines) and drain efficiency (blue squared lines) at $104 \mathrm{MHz}$. Solid lines refer to the transistor extrinsic plane, dotted lines to the PA connector plane.

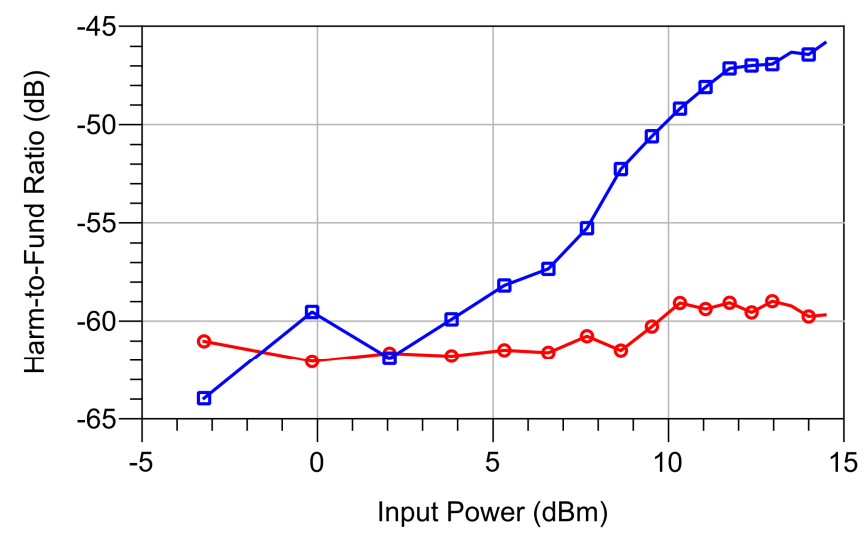

Fig. 13 Harmonic-to-fundamental output-power ratio for a fundamental frequency of $104 \mathrm{MHz}$.

TABLE V

Predicted ANd Measured TRANSISTOR PERformance AT 104 MHZ FOR THE CLASS-F PA

\begin{tabular}{ccc}
\hline \hline Nonlinear Embedding & Performance & $\begin{array}{c}\text { High-Frequency } \\
\text { Measurement }\end{array}$ \\
\hline $8.5 \mathrm{~W}$ & Output Power & $8.6 \mathrm{~W}$ \\
$86.7 \%$ & Drain Efficiency & $83.2 \%$ \\
\hline \hline
\end{tabular}

comparison, we measured the $\mathrm{S}$ parameters of the fabricated matching networks to shift the large-signal data at the transistor plane (see Fig. 8).

The S-parameters of the class-F output-matching-network showed a frequency shift with respect to the designed network due to fabrication issues, so that the best match with the theoretical load impedances is achieved at $104 \mathrm{MHz}$. The best performance at the transistor plane is reported in Table $\mathrm{V}$ compared with the prediction of the nonlinear-embedding procedure. The good agreement suggests the transistor is operating with the same load line we have measured at low frequency at the current-generator plane. In Fig. 12, we show the corresponding performance as a function of the input power.

It is also significant to observe the spectrum of the output power to monitor the harmonic distortion. In Fig. 13, we show the harmonic-to-fundamental output-power ratio for a fundamental frequency of $104 \mathrm{MHz}$ as a function of the input power. The second harmonic is practically absent in the output 


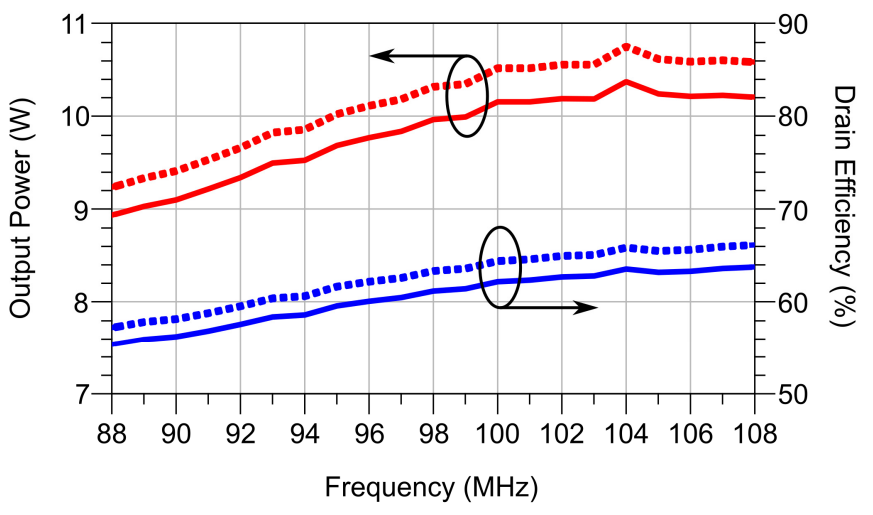

Fig. 14 Output power and drain efficiency for the class-AB PA for an input power of $17 \mathrm{dBm}(50 \mathrm{~mW})$. Dotted lines refer to the transistor extrinsic plane, solid lines to the PA connector plane.

\section{TABLE VI}

LOAD IMPEDANCES PREDICTED BY LINEAR AND NONLINEAR-EMBEDDING APPROACHES AT $100 \mathrm{MHz}$

\begin{tabular}{ccccc}
\hline \hline Class & Freq. & $\begin{array}{c}\text { Linear } \\
\text { Embedding A }\end{array}$ & $\begin{array}{c}\text { Linear } \\
\text { Embedding B }\end{array}$ & $\begin{array}{c}\text { Nonlinear } \\
\text { Embedding }\end{array}$ \\
\hline \multirow{2}{*}{$\mathrm{F}$} & $100 \mathrm{MHz}$ & $105+\mathrm{j} 67 \Omega$ & $101+\mathrm{j} 67.8 \Omega$ & $77.8+\mathrm{j} 71.1 \Omega$ \\
& $200 \mathrm{MHz}$ & $\mathrm{j} 6.5 \Omega$ & $\mathrm{j} 6.1 \Omega$ & $\mathrm{j} 2.0 \Omega$ \\
$\mathrm{AB}$ & $300 \mathrm{MHz}$ & $3.2+\mathrm{j} 79.7 \Omega$ & $0.8+\mathrm{j} 77.7 \Omega$ & $\mathrm{j} 59.7 \Omega$ \\
& $100 \mathrm{MHz}$ & $74.3+\mathrm{j} 13.2 \Omega$ & $71.9+\mathrm{j} 13.5 \Omega$ & $67.9+\mathrm{j} 20.6 \Omega$ \\
\hline \hline
\end{tabular}

spectrum, whereas there is a small third-harmonic component, which becomes evident only for the higher power levels. This result is indeed consistent, since one of the aims of the class-F operation is to minimize the output power at harmonics to improve the PA efficiency.

In the same way, we shifted the measured data at the transistor reference plane for the class-AB PA. We report the results in Fig. 14 over the FM bandwidth for a constant input power (i.e., $50 \mathrm{~mW}$ ). The achieved performance is slightly lower than the expected one in terms of efficiency. The analysis of the output-matching-network S-parameters showed that the terminations at harmonics presented at the DUT plane, which were not controlled, were higher than expected, which cause a slight detrimental effect on the PA efficiency.

\section{Validation and comparison with conventional design approaches}

As shown so far, the nonlinear-embedding methodology transposes the current-generator waveforms to the extrinsic plane by considering a full nonlinear model of the intrinsic capacitances and a complete linear parasitic network. This surely makes it more complicated than conventional but less accurate approaches usually based on a simplified linear approximation of the transistor nonidealities, can lead, as a consequence, to less accurate results. To provide some comparison, we applied two "linear" embedding procedures [47] starting from the class-F and class-AB load lines at the current-generator plane. For the first case (A), we only considered two linear capacitances, $C_{g s}$ and $C_{d s}$, as the capacitive core and no parasitic elements have been included to transpose the data at the extrinsic plane. For the second case (B), we also included the effects of the parasitic elements and

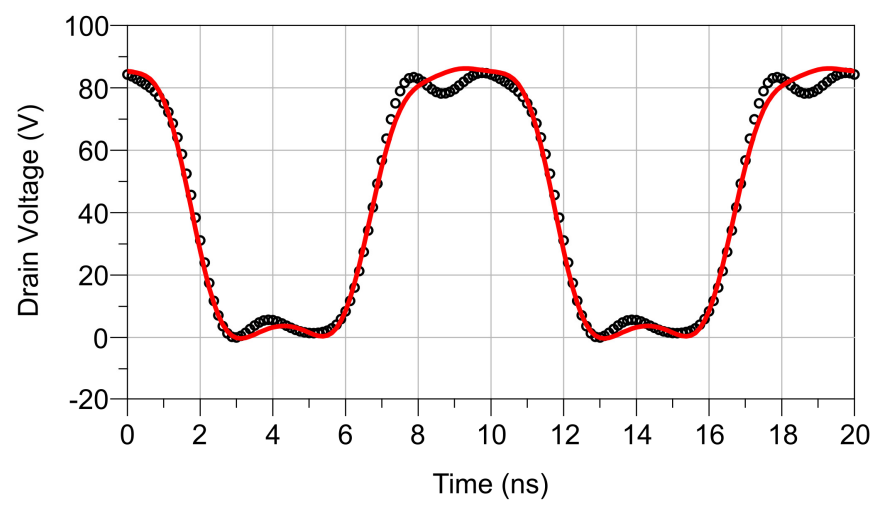

(a)

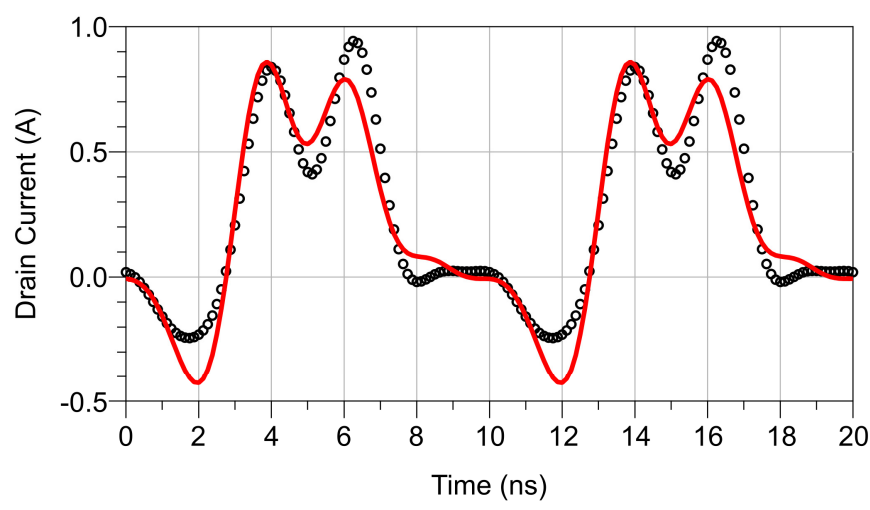

(b)

Fig. 15 Drain voltage (a) and drain current (b) waveforms at the transistor extrinsic plane for the class-F PA measured at $100 \mathrm{MHz}$ for an input power of $14.5 \mathrm{dBm}(28 \mathrm{~mW})$ : expected waveforms derived by the nonlinear embedding (solid lines) and measured waveforms (symbols).

the linear capacitance $C_{g d}$. In case A there is no feedback element between the input and output ports of the device. Such a practical hypothesis is commonly adopted [15],[19]-[21],[24] since it strongly simplifies the evaluation of the extrinsic load impedances, by reversely isolating the two ports of the device. In case $\mathrm{B}$ we introduced the capacitance $C_{g d}$ as a feedback element, in order to show that the limited accuracy is largely ascribable to the linearity hyphothesis.

In Table VI, we report results of the impedances predicted by the linear approaches in comparison with the nonlinear one. The data provided by the linear approaches are very similar, which confirms the small influence of the parasitic network and of the feedback capacitance $C_{g d}$. On the contrary, compared with the nonlinear embedding, we note a considerable difference, especially for the class-F condition, where the effects of the capacitance nonlinearities is more noticeable and strongly influence the transistor operation.

To furtherly confirm the validity of the nonlinear-embedding procedure, we characterized the transistor performance at the design frequency. Starting from the impedances derived at design frequency (see Tables III and IV), we can directly synthesize them at the extrinsic plane by means of a timedomain load-pull setup based on a 4-channel $50-\mathrm{GHz}$ oscilloscope [48], and compare the measured time-domain waveforms with the ones predicted by the nonlinear embedding. 
This comparison becomes very interesting for the class-F operation, since it involves some harmonic tuning up to the third harmonic. We report the results for a design frequency of $100 \mathrm{MHz}$ in Fig. 15. The main difference between predicted and measured waves is related to the limitation of synthesizing harmonic terminations with a theoretically zero real part, which increases the magnitude of the harmonics with respect to the ideal case. Despite this aspect, the agreement is very good and proves the accuracy of the predicted results.

\section{CONCLUSION}

In this paper, we applied the nonlinear-embedding technique [26] as a general approach for the design of LDMOS PAs. We designed and realized a high-efficiency class-F PA and a wideband class-AB PA operating in the FM broadcasting band. The performance of the fabricated PAs shows very good agreement with design predictions, proving the effectiveness of the proposed approach and paving the way for further development of the nonlinear-embedding approach oriented to LDMOS technology.

\section{ACKNOWLEDGMENT}

The Authors would like to thank ELENOS s.r.l. who partly supported this work.

\section{REFERENCES}

[1] A. I. Colli-Menchi and E. Sánchez-Sinencio, "A high-efficiency selfoscillating class-D amplifier for piezoelectric speakers," IEEE Trans. Power Electronics, vol. 30, no. 9, pp. 5125-5135, Sep. 2015.

[2] G. R. Walker, "A class B switch-mode assisted linear amplifier," IEEE Trans. Power Electronics, vol. 18, no. 6, pp. 1278-1285, Nov. 2003.

[3] V. Valente, C. Eder, N. Donaldson, and A. Demosthenous, "A high-power CMOS class-D amplifier for inductive-link medical transmitters," IEEE Trans. Power Electronics, vol. 30, no. 8, pp. 4477-4488, Aug. 2015.

[4] R. Paul, L. Sankey, L. Corradini, Z. Popovic, and D. Maksimovic, "Power management of wideband code division multiple access RF power amplifiers with antenna mismatch," IEEE Trans. Power Electronics, vol. 25, no. 4, pp. 981-991, Apr. 2010.

[5] M. Vasić, O. García, J. Á. Oliver, P. Alou, D. Diaz, R. Prieto, and J. A. Cobos, "Envelope amplifier based on switching capacitors for highefficiency RF amplifiers," IEEE Trans. Power Electronics, vol. 27, no. 3, pp. 1359-1368, Mar. 2012.

[6] S. C. Cripps, RF Power Amplifiers for Wireless Communication. Norwood, MA, USA: Artech House, 1999.

[7] P. Colantonio, F. Giannini, and E. Limiti, High Efficiency RF and Microwave Solid State Power Amplifiers. New York, NY, USA: Wiley, 2009.

[8] S. D. Kee, I. Aoki, A. Hajimiri, and D. Rutledge, "The class-E/F family of ZVS switching amplifiers," IEEE Trans. Microw. Theory Tech., vol. 51, no. 6, pp. 1677-1690, Jun. 2003.

[9] M. Hayati, A. Sheikhi, and A. Grebennikov, "Design and analysis of class E/F3 power amplifier with nonlinear shunt capacitance at nonoptimum operation," IEEE Trans. Power Electronics, vol. 30, no. 2, pp. 727-734, Feb. 2015.

[10] M. Hayati, A. Sheikhi, and A. Grebennikov, "Effect of nonlinearity of parasitic capacitance on analysis and design of class E/F3 power amplifier," IEEE Trans. Power Electronics, vol. 30, no. 8, pp. 4404-4411, Aug. 2015.

[11] T. Canning, P. J. Tasker, and S. C. Cripps, "Continuous mode power amplifier design using harmonic clipping contours: theory and practice," IEEE Trans. Microw. Theory Techn., vol. 62, no. 1, pp. 100-110, Jan. 2014.

[12] F. H. Raab, "Class-F power amplifiers with maximally flat waveforms," IEEE Trans. Microw. Theory and Tech., vol. 45, no. 11, pp. 2007-2012, Nov. 1997.
[13] C. Steinbeiser, P. Page, T. Landon, and G. Burgin, "Doherty power amplifiers using 2nd generation HVHBT technology for high efficiency basestation applications," IEEE Compound Semiconductor Integrated Circuit Symp., 2010, pp. 1-4.

[14] M.A. Belaïd, K. Ketata, M. Gares, K. Mourgues, M. Masmoudi, and J. Marcon, "Comparative analysis of RF LDMOS capacitance reliability under accelerated ageing tests," Microelectronics Reliability, vol. 47, no. 1, pp. 59-64, Jan. 2007.

[15] A. Mediano and N. O. Sokal, "A class-E RF power amplifier with a flattop transistor-voltage waveform," IEEE Trans. Power Electronics, vol. 28, no. 11, pp. 5215-5221, Nov. 2013.

[16] T. Suetsugu and M. K. Kazimierczuk, "Design procedure for lossless voltage-clamped class E amplifier with a transformer and a diode," IEEE Trans. Power Electronics, vol. 20, no. 1, pp. 56-64, Jan. 2005.

[17] V. Vadalà, A. Raffo, S. Di Falco, G. Bosi, A. Nalli and G. Vannini, "A load-pull characterization technique accounting for harmonic tuning," IEEE Trans. Microw. Theory Techn., vol. 61, no. 7, pp. 2695-2704, Jul. 2013.

[18] S. Preis, T. Arnous, Z. Zhang, P. Saad, and G. Boeck, "Bandwidth versus efficiency performance using power combining in GaN HEMT power amplifiers," 2013 Eur. Microw. Conf., 2013, pp. 696-699.

[19] K. Narendra and T. Yewkok, "Optimised high-efficiency class E radio frequency power amplifier for wide bandwidth and high harmonics suppression," IET Circuits, Devices \& Systems, vol. 8, no. 2, pp. 82-89, Mar. 2014.

[20] A. Yefymovych, V. Krizhanovski, R. Giofrè, and P. Colantonio, "Load network design technique for microwave class-F amplifier," 20th Int. Conf. Microw., Radar and Wireless Commun., 2014, pp. 1-3.

[21] P. Boontamchauy, R. Phudpong, K. Wongthavarawat and S. Chalermwisutkul, "Wideband class-E power amplifier with flat gain for FM radio transmitters," IEEE Conf. Antenna Measurements and Applications, 2015, pp. 1-4.

[22] M. Iqbal and A. Piacibello, "A $5 \mathrm{~W}$ class-AB power amplifier based on a GaN HEMT for LTE communication band," 16th Mediterranean Microw. Symp., 2016, pp. 1-4.

[23] M. Iqbal and A. Piacibello, "GaN HEMT based class-F power amplifier with broad bandwidth and high efficiency," 2016 Int. Conf. Integr. Circuits and Microsystems, 2016, pp. 131-134.

[24] R. Amirpour, R. Darraji, F. Ghannouchi, and R. Quay, "Enhancement of the broadband efficiency of a class-J power amplifier with varactor-based dynamic load modulation," IEEE Microw. Compon. Lett., vol. 27, no. 2, pp. 180-182, Feb. 2017.

[25] Y. Dong, L. Mao, and S. Xie, "Extended continuous inverse class-F power amplifiers with class-AB bias conditions," IEEE Microw. Compon. Lett., vol. 27, no. 4, pp. 368-370, Apr. 2017.

[26] A. Raffo, F. Scappaviva, and G. Vannini, "A new approach to microwave power amplifier design based on the experimental characterization of the intrinsic electron-device load line," IEEE Trans. Microw. Theory Techn., vol. 57, no. 7, pp. 1743-1752, Jul. 2009.

[27] S. L. Chen and Y. S. Lai, "Strengthen anti-ESD characteristics in an HV LDMOS with superjunction structures," IEEE Trans. Power Electronics, vol. 30, no. 5, pp. 2375-2382, May 2015.

[28] W. Du and X. Chen, "Design of a double-gate power LDMOS with improved SOA by complementary majority carrier conduction paths," IEEE Trans. Power Electronics, vol. 31, no. 7, pp. 5133-5140, Jul. 2016.

[29] X. Deng, X. Chen, D. Liu, K. Liang, Z. Gan, Z. Li, and B. Zhang, "An accumulation mode RF laterally double diffused MOSFET with improved performance," IEEE Electron Device Lett., vol. 37, no. 10, pp. 1321-1323, Oct. 2016.

[30] H. M. Nemati, C. Fager, M. Thorsell, and H. Zirath, "High-efficiency LDMOS power-amplifier design at $1 \mathrm{GHz}$ using an optimized transistor model," IEEE Trans. Microw. Theory Techn., vol. 57, no. 7, pp. 1647-1654, Jul. 2009.

[31] J. Cumana, A. Grebennikov, G. Sun, N. Kumar, and R. H. Jansen, "An extended topology of parallel-circuit class-E power amplifier to account for larger output capacitances," IEEE Trans. Microw. Theory Techn., vol. 59, no. 12, pp. 3174-3183, Dec. 2011.

[32] F. Hou and Y. Zhang, "RF LDMOS power transistor for multi-carrier GSM base station," IEEE Int. Wireless Symp, 2014, pp. 1-3.

[33] M. J. Pelk, W. C. E. Neo, J. R. Gajadharsing, R. S. Pengelly, and L. C. N. de Vreede, "A high-efficiency 100-W GaN three-way Doherty amplifier for base-station applications," IEEE Trans. Microw. Theory Techn., vol. 56, no. 7, pp. 1582-1591, Jul. 2008. 
[34] H. Jang, P. Roblin, and Z. Xie, "Model-based nonlinear embedding for power-amplifier design," IEEE Trans. Microw. Theory Techn., vol. 62, no. 9, pp. 1986-2002, Sep. 2014.

[35] B. J. Sheu, D. L. Scharfetter, P. K. Ko, and M. C. Jeng, "BSIM: Berkeley short-channel IGFET model for MOS transistors," IEEE J. Solid-State Circuits, vol. 22, no. 4, pp. 558-566, Aug. 1987.

[36] I. Angelov, H. Zirath, and N. Rorsman, "A new empirical nonlinear model for HEMT-devices," IEEE MTT-S Microwave Symp. Dig., 1992, pp. $1583-1586$.

[37] A. Raffo, V. Vadalà, D. M. M.-P. Schreurs, G. Crupi, G. Avolio, A. Caddemi, and G. Vannini, "Nonlinear dispersive modeling of electron devices oriented to GaN power amplifier design," IEEE Trans. Microw. Theory Techn., vol. 58, no. 4, pp. 710-718, Apr. 2010.

[38] D. E. Root, "Nonlinear charge modeling for FET large-signal simulation and its importance for IP3 and ACPR in communication circuits," Proc. 44th IEEE 2001 Midwest Sympo. Circuits Syst., 2001, pp. 768-772.

[39] G. Crupi, D. M. M.-P. Schreurs, A. Caddemi, A. Raffo, and G. Vannini, "Investigation on the non-quasi-static effect implementation for millimeter-wave FET models," Int. J. RF and Microwave Comp. Aid. Eng., vol. 20, no. 1, pp. 87-93, Jan. 2010.

[40] G. Avolio, D. M. M. P. Schreurs, A. Raffo, G. Crupi, G. Vannini, and B. Nauwelaers, "Waveforms-only based nonlinear de-embedding in active devices," IEEE Microw. Compon. Lett., vol. 22, no. 4, pp. 215-217, Apr. 2012.

[41] G. Bosi, A. Raffo, G. Vannini, E. Cipriani, P. Colantonio and F. Giannini, "Gate waveform effects on high-efficiency PA design: An experimental validation," 9th Eur. Microw. Integr. Circuit Conf., 2014, pp. 329-332.

[42] A. Raffo, V. Vadalà, G. Bosi, F. Trevisan, G. Avolio, and G. Vannini, "Waveform engineering: state-of-the-art and future trends," Int. J. RF Microw. Comput. Aided Eng., vol. 27, no. 1, Jan. 2017, e21051.

[43] A. Raffo, S. D. Falco, V. Vadalà, and G. Vannini, "Characterization of GaN HEMT low-frequency dispersion through a multiharmonic measurement system," IEEE Trans. Microw. Theory Techn., vol. 58, no. 9, pp. 2490-2496, Sep. 2010

[44] G. Bosi, G. Crupi, V. Vadalà, A. Raffo, A. Giovannelli, and G. Vannini, "Nonlinear modeling of LDMOS transistors for high-power FM transmitters," Int. J. Numer. Model., vol. 27, no. 5-6, pp. 780-791, Sep.-Dec. 2014.

[45] J. Stenarson and K. Yhland, "A reformulation of TRL and LRM for Sparameters," 73rd ARFTG Microwave Measurement Conf., 2009, pp. $1-4$.

[46] G. Crupi, D. M. M.-P. Schreurs, A. Raffo, A. Caddemi, and G. Vannini, "A new millimeter-wave small-signal modeling approach for pHEMTs accounting for the output conductance time delay," IEEE Trans. Microw. Theory Techn., vol. 56, no. 4, pp. 741-746, Apr. 2008.

[47] A. Raffo, V. Vadalà, G. Avolio, G. Bosi, A. Nalli, D. M. M.-P. Schreurs, and G. Vannini, "Linear versus nonlinear de-embedding: experimental investigation," 81st ARFTG Microwave Measurement Conf., 2013, pp. $1-5$.

[48] D. A. Humphreys, A. Raffo, G. Bosi, G. Vannini, D. Schreurs, K. N. Gebremicael, and K. Morris, "Maximizing the benefit of existing equipment for nonlinear and communication measurements," 87th ARFTG Microwave Measurement Conf., 2016, pp. 1-4.

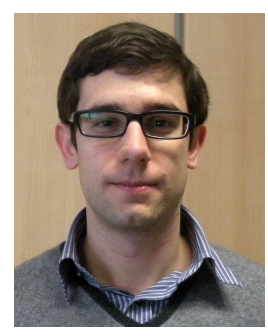

Gianni Bosi (S'11-M'15) was born in Copparo (FE), Italy, in 1986. He received the M.S. degree (with honors) in engineering and technologies for telecommunications and electronics and $\mathrm{PhD}$ degree in information engineering from the University of Ferrara, Ferrara, Italy, in 2010 and 2014 respectively. Since 2011, he has been with the Engineering Department, University of Ferrara. His research activity is mainly oriented to nonlinear characterization and modeling of microwave EDs, HMICs and MMIC design.

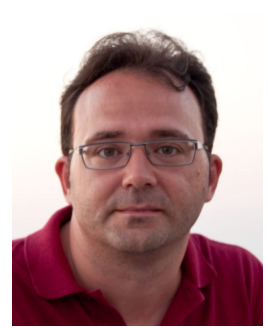

Antonio Raffo (S'04-M'07) was born in Taranto, Italy, in 1976. He received the M.S. degree (Hons.) in electronic engineering and Ph.D. degree in information engineering from the University of Ferrara, Ferrara, Italy, in 2002 and 2006, respectively.

Since 2002, he has been with the Engineering Department, University of Ferrara, where he is currently a Research Associate and teaches courses in semiconductor devices and electronic instrumentation and measurement. He has co-authored over 120 publications in international journals and conferences and co-edited Microwave Wireless Communications: From Transistor to System Level (Elsevier, 2016). His current research interests include nonlinear electron device characterization and modeling and circuit-design techniques for nonlinear microwave and millimeter-wave applications.

Dr. Raffo is a member of the Technical Program Committee of the IEEE International Workshop on Integrated Nonlinear Microwave and Millimetrewave Circuits (INMMiC) and the IEEE Microwave Measurement Technical Committee. He serves as an Associate Editor of the International Journal of Numerical Modeling: Electronic Networks, Devices, and Fields, and was the Technical Program Committee Chair of the IEEE INMMiC Conference, Leuven, Belgium, in 2014.

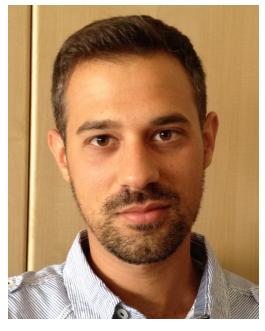

Francesco Trevisan was born in Rovigo, Italy, in 1988. He received the M.S. degree in engineering and technologies for telecommunications and electronics from the University of Ferrara, Ferrara, Italy, in 2013, and is currently working toward the Ph.D. degree at the University of Ferrara. Since 2014, he has been with the Engineering Department, University of Ferrara. His research activity is mainly oriented to nonlinear device and circuit characterization and modeling for microwave applications.

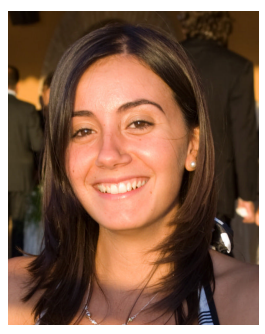

Valeria Vadalà (S'07-M'11) was born in Reggio Calabria, Italy, in 1982. She received the $\mathrm{M}$. S. degree (with honors) in electronic engineering from the "Mediterranea" University of Reggio Calabria, Reggio Calabria, Italy, in 2006 and the Ph.D. degree in information engineering from the University of Ferrara, Ferrara, Italy, in 2010. Dr. Vadalà is currently with the Department of Engineering, University of Ferrara, as a Post- 
Doctoral Researcher. Her current research interests include nonlinear electron-device characterization and modeling and circuit-design techniques for nonlinear microwave and millimeter-wave applications.

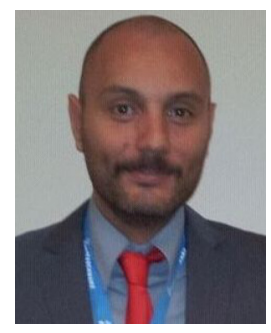

Giovanni Crupi (S'04-M'12-SM'13) received the MSc in electronic engineering (cum laude) and the $\mathrm{PhD}$ degrees from the University of Messina, Italy, in 2003 and in 2006, respectively.

Currently, he is an Associate Professor with the University of Messina, Italy, where he has taught/teaches bioengineering, microwave electronics, laboratory of wireless technologies, and optoelectronics. Since 2005, he has been a repeat visiting scientist with KU Leuven and IMEC, Leuven, Belgium. His main research interests include small and large signal modeling of advanced microwave devices. He has authored or coauthored around 150 publications in international journals and conferences. He has coedited two books entitled "Microwave De-embedding: From Theory to Applications" (Academic Press, Oxford, UK, 2013) and "Microwave Wireless Communications: From Transistor to System Level" (Academic Press, Oxford, UK, 2016).

Dr. Crupi serves as an Associate Editor of the International Journal of RF and Microwave Computer-Aided Engineering and the International Journal of Numerical Modelling: Electronic Networks, Devices and Fields. He serves as TPC Member of the IEEE INMMiC and TELSIKS conferences and was also TPC Chair of the IEEE INMMiC in 2014 and 2015. Dr. Crupi is a Senior Member of the IEEE and the Chair of the IEEE Microwave Theory and Techniques Society (MTT-S) Fellowship program.

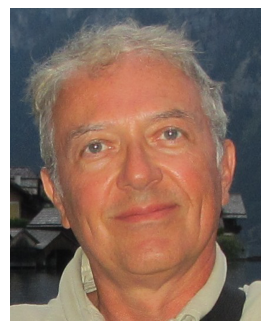

Giorgio Vannini (S'87-M'92) received the Laurea degree in electronic engineering and $\mathrm{Ph}$. D. degree in electronic and computer science engineering, from the University of Bologna, Bologna, Italy. In 1992, he joined the Department of Electronics, University of Bologna, as a Research Associate. From 1994 to 1998, he was with the Research Centre on Electronics, Computer science and Telecommunication Engineering, National Research Council (CSITE), Bologna, Italy, where he was responsible for MMIC testing and the Computer-Aided Design (CAD) Laboratory. In 1998, he joined the University of Ferrara, Ferrara, Italy, as an Associate Professor, and since 2005, as a Full Professor of electronics. He was Head of the Engineering
Department from 2007 to 2015 and Member of the Board of Directors from 2010 to 2012. During his academic career he has coauthored over 250 articles devoted to electron device modeling, computer aided design techniques for MMICs, and nonlinear circuit analysis and design. He is a cofounder of the academic spinoff Microwave Electronics for Communications (MEC) and a member of the Technical Scientific Committee of the "MIST E-R" Laboratory for Industrial Research and Technology Transfer. 